\title{
Right Ovary
}

National Cancer Institute

\section{Source}

National Cancer Institute. Right Ovary. NCI Thesaurus. Code C33487.

The ovary that is located in the right side of the pelvis. 